\title{
Análisis de variables e indicadores empleados para medir la sociedad de la información
}

\author{
Analysis of variables and indicators used to measure the information society
}

\author{
Mayda Patricia González-Zabala1 $\quad$ Jenny Marcela Sánchez-Torres² \\ Recibido 11 de noviembre de 2011, aceptado 13 de agosto de 2012 \\ Received: November 11, $2011 \quad$ Accepted: August 13, 2012
}

\begin{abstract}
RESUMEN
El propósito de este artículo es presentar los resultados de una investigación que consistió en caracterizar sistemas de evaluación de la Sociedad de la Información -SI- propuestos en el período de 1995 a 2010 a través del análisis de sus variables e indicadores. Para lograr los resultados se definió una metodología consistente en cuatro etapas: i) identificación de las propuestas de evaluación de la SI; ii) selección de las propuestas a ser analizadas; iii) identificación y clasificación de variables e indicadores; y iv) generación de informes y análisis de resultados. Los principales resultados fueron: identificar que el 37,3\% de las variables/indicadores en estudio se enfocan en medir aspectos de infraestructura, en cuanto que un $3,1 \%$ están enfocados en la medición de la participación de los ciudadanos en la SI. De igual manera, se identificó que la medición del avance de la SI considera aspectos relacionados con el contexto y que la oferta de variables/indicadores se ha ampliado a medir diversas áreas de la sociedad tales como: el turismo, la ciencia, la agronomía, etc. Por último, este trabajo permite analizar en detalle los aspectos que son considerados en la medición de la SI, así como el número de variables/indicadores propuestos en cada uno de ellos.
\end{abstract}

Palabras clave: Indicadores, medición, sistemas de medición, sociedad de la información, variables.

\begin{abstract}
The purpose of this paper is to present a characterization of the evaluation systems of Information Society (IS) proposed in the period 1995 to 2010, based on an analysis of their variables and indicators. To obtain the results, a methodology composed of four stages was defined: i) identification of the evaluation systems of IS; ii) selection of the evaluation systems for the analysis; iii) identification and classification of variables and indicators; and iv) generation of reports and analysis of results. The main results show that $37.3 \%$ of variables/indicators under study are focused on infrastructure aspects, and, $3.1 \%$ are focused on measuring the participation of citizens in the IS. Similarly, it is found out that measuring the progress of the IS considers issues related to the context and the amount of variables / indicators has been extended to measure several areas of society such as: tourism, science, agronomy, etc. Last, this work allows to analyze in detail the aspects that are considered in the measurement of IS, and the number of variables/indicators proposed in each of them.
\end{abstract}

Keywords: Indicators, measurement, measurement systems, information society, variables.

\footnotetext{
1 Facultad de Ingeniería. Universidad del Magdalena. Carrera 32 No 22-08. Santa Marta, Colombia. E-mail: mpgonzalez@unimagdalena.edu.co

2 Departamento de Ingeniería de Sistemas e Industrial. Universidad Nacional de Colombia. Carrera $45 \mathrm{~N}^{\circ}$ 26-85-Edificio Uriel Gutiérrez. Bogotá, Colombia. E-mail: jmsanchezt@unal.edu.co
} 


\section{INTRODUCCIÓN}

La Sociedad de la Información (SI) ha sido definida como un modelo que se apoya en la implantación de Tecnología de la Información y ComunicaciónTIC en todos los ámbitos, y en su uso adecuado, para hacer de la información y el conocimiento los motores del crecimiento productivo y el desarrollo económico y social [1]. Por lo cual, los diferentes países han incluido en sus agendas la promoción de este modelo de sociedad como una estrategia para el desarrollo, progreso y prosperidad. Además, con las estrategias de desarrollo de la SI se pretende superar las brechas sociales, económicas y oportunidades de la población [1-2], y generar las condiciones necesarias para que la incorporación de la SI sea posible [3]. Del mismo modo, se han generado esfuerzos conjuntos a nivel supranacional y regional con el fin de crear planes que respondan a las necesidades y realidades de cada región [4-8].

Por otro lado, la necesidad de evaluar la incorporación de la SI se expresa en la "Agenda de Túnez para la Sociedad de la Información" [9] y el "Compromiso de Túnez" [10]. De esta manera, los organismos supranacionales y nacionales se han interesado en la medición de su progreso. Para este fin se han desarrollado varios sistemas de medición internacionales, los cuales permiten la comparación en el tiempo y entre regiones, además de proveer insumos para analizar los resultados obtenidos y hacer ajustes a las políticas implementadas [1112]; muchos de estos sistemas son utilizados como referencia en diferentes países. Asimismo, la mayoría de estos sistemas de medición tienen como salida un indicador sintético, que es el resultado del cálculo de las variables e indicadores ${ }^{3}$ que componen cada sistema.

Al considerar la importancia de la SI y que su medición es relevante y necesaria para establecer los avances obtenidos y las políticas de fortalecimiento,

\footnotetext{
3 Una variable es un concepto de estudio que puede asumir valores diferentes de los comprendidos en un conjunto (Diccionario Espasa, 1997 citado en [1]. Y un indicador es una característica observable y claramente identificable de una variable. La variable denota un aspecto que desea medirse de un objeto de estudio y para conseguirlo se establece un conjunto de indicadores claramente definidos que permiten asignarle a la variable un determinado valor [1]
}

cabe preguntar ¿qué aspectos se evalúan para determinar el estado de la SI? Para responder a esta pregunta, se realizó una investigación centrada en el análisis de las variables e indicadores de los sistemas de evaluación de la SI propuestos en el período de 1995 a 2010 con el fin de determinar los aspectos contemplados en el proceso evaluativo. Dicha investigación se realizó en el marco del desarrollo de la tesis doctoral titulada "Modelo para evaluar la e-inclusión. El caso colombiano", financiada por el Departamento Administrativo de Ciencia y Tecnología de Colombia-COLCIENCIAS [13-14], la cual hace parte del proyecto e-métrica Colombia desarrollado por la Universidad Nacional de Colombia-Fase I [15].

\section{METODOLOGÍA}

Para realizar el análisis de las variables e indicadores que componen cada sistema de evaluación se definieron cuatro etapas, basadas en el trabajo realizado por [1], las cuales se describen a continuación:

\section{Etapa 1: Identificación de propuestas de evaluación de la SI}

En esta etapa se identificaron las diferentes propuestas de evaluación de la SI (sistemas y guías), propuestas por entidades supranacionales y nacionales en el período 1995-2010. Como punto de partida se tomó el trabajo desarrollado por [1] y [16]. Posteriormente, la información fue verificada y complementada, para lo cual se consultaron los informes y reportes oficiales disponibles de cada uno de los sistemas detectados. Asimismo se realizó una nueva búsqueda de información orientada a detectar sistemas de evaluación que no estuvieran detectados por [1] y [16].

\section{Etapa 2: Selección de las propuestas (sistemas y directrices) de evaluación}

En esta etapa se seleccionaron las propuestas de evaluación de la SI que serían analizadas. Los criterios de selección que se tomaron en consideración fueron:

- la información disponible: se buscaron fuentes de información primaria y secundaria de cada uno de los sistemas; aquellos sistemas que no fue posible encontrar documentación fueron descartados. 
- $\quad$ y que la evaluación no se realizara en aspectos específicos de la SI: se seleccionaron solo sistemas de medición que evaluaran de forma general los avances de la SI, por ejemplo, los indicadoresKEI Economía del Conocimiento propuesta por la Comisión Europea [17] fueron descartados debido a que sólo se centran en los aspectos económicos de la SI. Es importante aclarar que los sistemas descartados fueron posteriormente analizados y tomados como punto de partida en el desarrollo del proyecto e-métrica [15], el cual busca crear un marco de medición para cada uno de los sectores de la SI.

\section{Etapa 3: Identificación y clasificación de las variables e indicadores}

En esta sección se identificaron las variables e indicadores de cada sistema de evaluación seleccionados. Posteriormente se clasificaron en las dimensiones de análisis y en sus aspectos específicos, los cuales fueron determinados tomando en consideración la definición de la SI y el análisis de los de modelos conceptuales de SI propuestos por [1], [3] y [18], los cuales definen los elementos esenciales de la SI.

A continuación se especifican las dimensiones de análisis y sus aspectos relacionados:

- Infraestructura: son los elementos o servicios esenciales para la operación y/o creación de productos de Tecnologías de la Información-TI. Los aspectos definidos para esta dimensión son: elementos y servicios físicos, servicios digitales, servicios y sectores relacionados con las TIC.

- Contexto: se trata de las condiciones externas que son necesarias para el desarrollo de la SI. Sus aspectos son: Marcos regulatorios; investigación, desarrollo e incentivos; capital humano; formación digital; aspectos de la economía; y acceso básico-oportunidad.

- E-sectores: son los sectores de la sociedad que cuentan con el apoyo de TI y servicios digitales. Los aspectos definidos para esta dimensión son: e-learning, comercio electrónico (e-commerce), e-salud, e-banca, e-multimedia, gobierno electrónico, e-cultura, e-arte, e-ciencia, e-turismo, y otras áreas.
- Uso: son los aspectos relacionados con el empleo de productos y servicios digitales y de TI.

- La participación en la sociedad en la SI: enfocada al acceso y uso de servicios de la SI, así como el aprovechamiento de sus beneficios. Los aspectos de análisis de esta dimensión son: e-inclusión, e-accesibilidad, e-competencias y brecha digital.

\section{Etapa 4: Generación de informes y análisis de resultados}

En esta etapa se generaron varios reportes con el fin de analizar las variables e indicadores, categorizadas con base en las dimensiones y los aspectos definidos. Para realizar los reportes se contó con una aplicación de software desarrollado para apoyar la clasificación y análisis de las variables y los indicadores [19]. El análisis se centró en:

- Distribución en las dimensiones propuestas con anterioridad.

- Distribución de variables e indicadores en cada dimensión.

- Distribución en los sistemas de evaluación seleccionados.

- Por dimensión y por aspecto.

\section{RESULTADOS}

En esta sección se presentan los resultados obtenidos en cada una de las etapas del proceso investigativo.

\section{Etapa 1: Identificación de propuestas de evaluación de la SI}

En esta etapa se identificaron 72 propuestas de evaluación del SI (67 del trabajo de [16] y cinco más en la búsqueda de verificación de información.

\section{Etapa 2: Selección de las propuestas (sistemas $y$ directrices) de referencia}

$\mathrm{Al}$ aplicar los criterios de selección establecidos se descartaron 51 sistemas de referencia, cinco de ellos por la dificultad de acceder a la información, 45 sistemas por evaluar aspectos específicos de la SI y uno de ellos para evitar duplicidad de información. En total se seleccionaron 21 sistemas de referencia, los cuales se presentan en la Tabla 1. 
Tabla 1. Listado de sistemas de evaluación seleccionados.

\begin{tabular}{|c|}
\hline Nombre \\
\hline $\begin{array}{l}\text { Annual Information Society Report [20], [21], [22], } \\
\text { [23] }\end{array}$ \\
\hline Connectivity Scorecard [24], [25], [26] \\
\hline Digital Opportunity Index - DOI [27]. [28] \\
\hline $\begin{array}{l}\text { eEspana [29], [30], [31], [32], [33], [34], [35], [36], } \\
\text { [37] }\end{array}$ \\
\hline $\begin{array}{l}\text { Europe's Digital Competitiveness Report Main } \\
\text { achievements of the i2010 strategy 2005-2009 [38] }\end{array}$ \\
\hline $\begin{array}{l}\text { European Survey of Information Society Projects } \\
\text { and Actions -[39], ESIS [40] }\end{array}$ \\
\hline $\begin{array}{l}\text { IDC's Information Society Index - ISI [41 ], [42 ], } \\
\text { [43] }\end{array}$ \\
\hline Indicadores de TIC [44] \\
\hline Indice de Adelanto Tecnológico IAT [45] \\
\hline $\begin{array}{l}\text { Information and communication technology } \\
\text { development indices [46] }\end{array}$ \\
\hline Information Economy Report [47] \\
\hline Knowledge Economy Index - KEI [17] \\
\hline OECD Key ICT Indicators [48] \\
\hline Sistema de información estadístico de TIC [49] \\
\hline State New Economy Index [50], [51] \\
\hline Digital Access Index -DAI [52] \\
\hline $\begin{array}{l}\text { Benchmarking Digital Europe 2011-2015 -A } \\
\text { conceptual framework [53] }\end{array}$ \\
\hline $\begin{array}{l}\text { Guide to Measuring the Information Society [54], } \\
\text { [55] }\end{array}$ \\
\hline $\begin{array}{l}\text { i2010 Information Space Innovation \& Investment } \\
\text { in } R \& D \text { Inclusion [56] }\end{array}$ \\
\hline $\begin{array}{l}\text { Indicadores clave de las tecnologías de la } \\
\text { información y de las comunicaciones [57] }\end{array}$ \\
\hline $\begin{array}{l}\text { The Global Information Society: a Statistical View } \\
\text { [58] }\end{array}$ \\
\hline
\end{tabular}

Fuente: [59-60]

\section{Etapa 3: Identificación y clasificación de las variables e indicadores}

En esta etapa, las variables y los indicadores de cada uno de los sistemas seleccionados fueron identificados, lo cual permitió obtener 782 variables e indicadores asociados a los 21 sistemas seleccionados. Seguidamente, se procedió a clasificar las variables e indicadores en las dimensiones y en los aspectos de análisis. Esta actividad permitió determinar que algunos de ellos eran susceptibles de ser clasificados en más de un aspecto. Esto permitió aumentar el número de variables e indicadores, pasando de 782 a 1010

\section{Etapa 4: Generación de informes}

A continuación se presentan los hallazgos encontrados en los análisis realizados, conforme a los análisis mencionados en la metodología.

\section{ESTUDIO DE VARIABLES E INDICADORES}

\section{Distribución de las variables/indicadores de las dimensiones de análisis}

Una vez se realizó la distribución de las variables e indicadores en las dimensiones, se determinó que la dimensión de la infraestructura tiene $37,3 \%$ de estos. Las dimensiones de e-sectores y contexto tienen un $29,9 \%$ y un $22,4 \%$ de las variables/indicadores, respectivamente. Por su parte, la dimensión de uso tiene un 7,3\% de las variables/indicadores y la dimensión de participación en la SI tiene $3,1 \%$ de las variables/indicadores.

En la Figura 1 se presenta la distribución en cada una de las dimensiones.

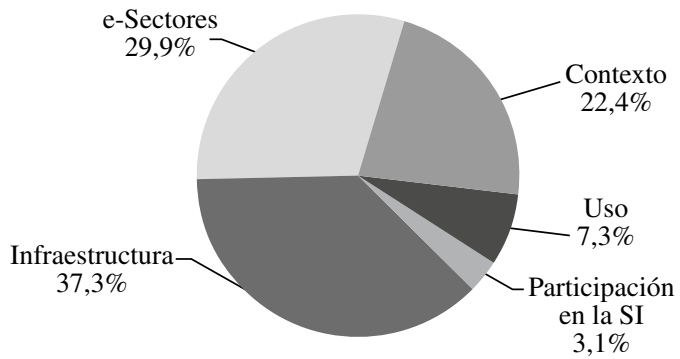

Figura 1. Distribución de variables/indicadores en las dimensiones de análisis

\section{Distribución de variables e indicadores en cada dimensión}

En este análisis se encontró que las variables se distribuyen como sigue: $27,5 \%$ en la dimensión de la infraestructura; el 33,6\% en e-sectores; $27,3 \%$ en contexto; $6,9 \%$ en el uso y el $4,7 \%$ en la dimensión de participación en la SI. Relacionados con los indicadores, el estudio muestra que están distribuidos de la siguiente manera: $44,4 \%$ en infraestructura; $27,2 \%$ en e-sectores; $18,9 \%$ en contexto; $7,7 \%$ en uso y el 1,9\% en la dimensión de participación en la SI. En total, los indicadores son 58,2\% del total y las variables son el $41,8 \%$. La Figura 2 presenta las dimensiones y su composición de variables e indicadores. 


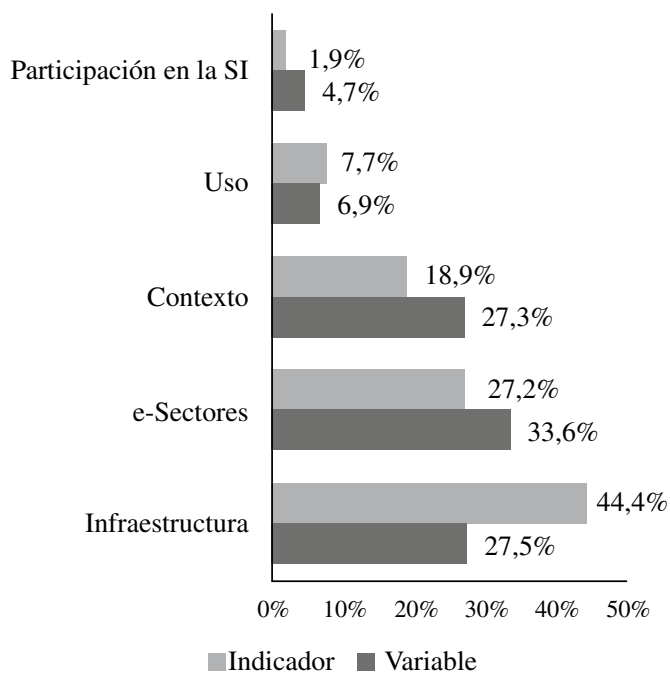

Figura 2. Distribución de variables e indicadores en cada dimensión.

\section{Distribución de las variables / indicadores en los sistemas de evaluación seleccionados}

Este análisis permitió identificar que seis de los 21 sistemas en estudio $(28,6 \%)$ tienen variables/ indicadores en cada dimensión propuesta; cinco sistemas $(23,8 \%)$ trabajan cuatro dimensiones, tres sistemas $(14,3 \%)$ involucran tres dimensiones y siete sistemas $(23,8 \%)$ evalúan dos dimensiones. Todos los sistemas de referencia tienen variables/indicadores relacionados con la dimensión de contexto. Los aspectos de infraestructura son evaluados por 20 sistemas (95\%). Las dimensiones de participación social y el uso fueron consideradas por 7 y 11 sistemas respectivamente, lo cual equivale al $33 \%$ y al 52\% de estos.

La Tabla 2 muestra la distribución de variables/ indicadores en los sistemas de evaluación seleccionados.

Tabla 2. Distribución de variables/indicadores en los sistemas de evaluación seleccionados.

\begin{tabular}{|l|c|c|c|c|c|c|}
\hline Referentes de Evaluación & Entorno & e-sector & Infrac. & Parti. & Uso & Total \\
\hline Annual Information Society Report & 11 & 21 & 12 & 7 & 2 & 53 \\
\hline $\begin{array}{l}\text { Benchmarking Digital Europe 2011-2015 - A } \\
\text { conceptual framework }\end{array}$ & 20 & 58 & 13 & 3 & 5 & 99 \\
\hline Connectivity Scorecard & 5 & 19 & 27 & 2 & 1 & 54 \\
\hline Digital Opportunity Index (DOI) & 2 & 0 & 12 & 0 & 0 & 14 \\
\hline eEspaña & 44 & 103 & 51 & 7 & 15 & 220 \\
\hline $\begin{array}{l}\text { Europe's Digital Competitiveness Report Main } \\
\text { achievements of the i2010 strategy 2005-2009 }\end{array}$ & 11 & 8 & 14 & 7 & - & 47 \\
\hline $\begin{array}{l}\text { European Survey of Information Society Projects } \\
\text { and Actions -ESIS }\end{array}$ & 1 & 1 & 18 & 0 & 0 & 20 \\
\hline Guide to Measuring the Information Society & 13 & 14 & 13 & 2 & 0 & 42 \\
\hline $\begin{array}{l}\text { i2010 Information Space Innovation \& Investment } \\
\text { in R\&D Inclusion }\end{array}$ & 16 & 20 & 15 & 3 & 1 & 56 \\
\hline IAT-Indice de Adelanto Tecnológico & 6 & & 4 & 0 & 0 & 10 \\
\hline IDC's Information Society Index (ISI) & 6 & 2 & 14 & 0 & 2 & 24 \\
\hline $\begin{array}{l}\text { Indicadores clave de las tecnologías de la } \\
\text { información y de las comunicaciones }\end{array}$ & 12 & 14 & 44 & 0 & 13 & 83 \\
\hline Indicadores de TIC & 1 & 0 & 0 & 0 & 7 & 3 \\
\hline $\begin{array}{l}\text { Information and Communication Technology } \\
\text { Development Indices }\end{array}$ & 4 & 0 & 13 & 0 & 0 & 17 \\
\hline Information Economy Report & 13 & 9 & 8 & 0 & 4 & 34 \\
\hline Knowledge Economy Index (KEI) & 9 & 0 & 3 & 0 & 0 & 12 \\
\hline OECD Key ICT Indicators & 18 & 2 & 25 & 0 & 0 & 45 \\
\hline Sistema de información estadístico de TIC & 3 & 0 & 7 & 0 & 0 & 10 \\
\hline State New Economy Index & 15 & 4 & 3 & 0 & 0 & $? ?$ \\
\hline The Digital Access Index (DAI) & 5 & 0 & 7 & 0 & 0 & 12 \\
\hline The Global Information Society: a Statistical View & 11 & 27 & 74 & 0 & 21 & 133 \\
\hline Total general & $\mathbf{2 2 6}$ & $\mathbf{3 0 2}$ & $\mathbf{3 7 7}$ & $\mathbf{3 1}$ & $\mathbf{7 4}$ & $\mathbf{1 0 1 0}$ \\
\hline
\end{tabular}

Fuente: [58-59]. 
Por otra parte, en la Tabla 2 se puede apreciar que el sistema de evaluación eEspaña es el que mayor número de variables/indicadores ha propuesto (220 equivalente al 21,8\% del total), asimismo éstos se encuentran distribuidos en todas las dimensiones propuestas en el presente estudio, donde se destaca el número de variables/indicadores propuestos para medir los diferentes e-sectores de la SI. De igual manera se destaca la continuidad que dicho sistema ha tenido, desde el año 2001, en la medición del avance de la SI en el contexto español.

Otros sistemas destacados, los cuales agrupan aproximadamente el 30\% del total de variables/ indicadores y aportan en todas las dimensiones, son: The Global Information Society: a Statistical View con el 13,2\% de las variables/indicadores, Benchmarking Digital Europe con el 9,8\% y el i2010 Information Space Innovation \& Investment in $R \& D$ Inclusion con el 5,5\% del total de variables/indicadores.

En relación con los sistemas a los cuales se les detectó menor número de variables/indicadores se encuentran: el Digital Opportunity Index (DOI), Indicadores de TIC y el IAT-Índice de Adelanto Tecnológico, los cuales aportan el 2,8\% del total de las variables/indicadores, además de centrarse en aspectos de infraestructura y no aportar en aspectos de participación y uso.

\section{Análisis de la dimensión infraestructura}

Este análisis se centra en la distribución de las variables e indicadores en cada uno de los aspectos que componen la dimensión. Los resultados muestran que el 51,5\% de las variables/indicadores se concentra en el aspecto de elementos y servicios físicos, el cual se asocia a la existencia de la tecnología. El aspecto de servicios digitales, el cual hace referencia a los servicios de Internet, software y telecomunicaciones, entre otros, tiene $26,8 \%$ de las variables/indicadores. El aspecto sector de TIC que está relacionado con el comercio de bienes, la competencia, las relaciones y los problemas de crecimiento tiene el $17,8 \%$ de las variables/indicadores de la dimensión. El aspecto servicios relacionados cuenta con $4 \%$ de las variables/indicadores, este aspecto asocia los servicios de mantenimiento, calidad y compras, entre otros. En la Figura 3 se muestra la distribución de las variables/indicadores en cada aspecto de la dimensión de la infraestructura.

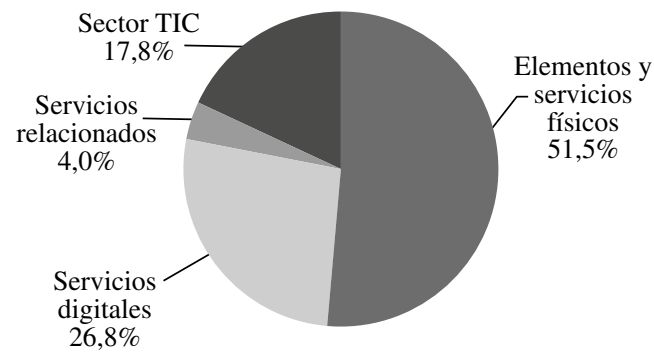

Figura 3. Distribución de variables/indicadores en cada aspecto de la dimensión infraestructura.

Posteriormente, al revisar la distribución de las variables y los indicadores en los aspectos de la dimensión de infraestructura se encontró que las variables se distribuyen como sigue: $43,1 \%$ en el aspecto de elementos y servicios físicos, $23,3 \%$ en servicios digitales, $23,3 \%$ en servicios relacionados y $10,3 \%$ en el sector de las TIC. Por otra parte, los indicadores se distribuyen en los aspectos así: 55,2\% en elementos y servicios físicos, el $28,4 \% \%$ en servicios digitales, $15,3 \%$ en servicios relacionados y el 1,1\% en el sector de las TIC. La Figura 4 muestra la distribución de las variables y los indicadores en cada uno de los aspectos de infraestructura.

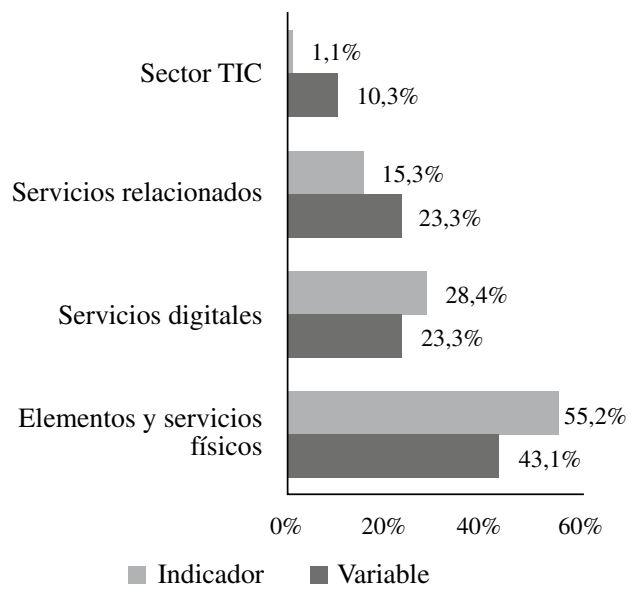

Figura 4. Distribución de variables e indicadores en los aspectos de la dimensión infraestructura.

\section{Análisis de la dimensión contexto}

En esta dimensión, la distribución de las variables en los aspectos fue: $32,2 \%$ en investigación; desarrollo e innovación; $23 \%$ en marco regulatorio; $18,1 \%$ en aspectos de economía; 9,3\% en financiación, 
inversión e incentivos; $5,3 \%$ en acceso básicooportunidad; $4,9 \%$ en formación digital, y $6,2 \%$ en capital humano. La Figura 5 muestra la distribución de la dimensión.

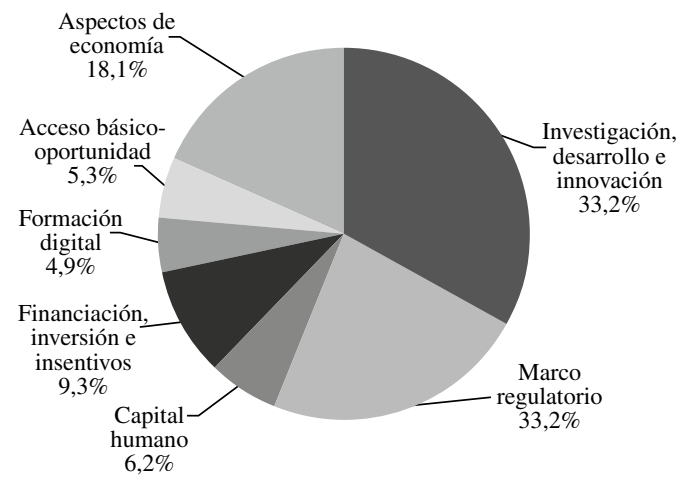

Figura 5. Distribución en cada uno de aspectos de la dimensión contexto.

Asimismo, al revisar la distribución de variables e indicadores que componen los aspectos de esta dimensión se detectó que las variables se distribuyen así: 33,9\% en investigación, desarrollo e innovación; $13,9 \%$ en marco regulatorio; $20 \%$ en aspectos de la economía; 8,7\% en acceso básico-oportunidad; $10,4 \%$ en formación digital y 5,2\% en capital humano. En la Figura 6 se presenta la composición de variables e indicadores de la dimensión en estudio.

Por otro lado, el $81 \%$ de los indicadores se concentran así: $32,4 \%$ en los aspectos relacionados con la investigación, desarrollo e innovación; 32,4\% en marco regulatorio y $16,2 \%$ en aspectos de economía. Los indicadores restantes se distribuyeron en los aspectos relacionados con el financiamiento, la inversión y los incentivos (10,8\%), capital humano $(3,6 \%)$ y formación digital $(4,5 \%)$. Ningún indicador fue asociado al aspecto acceso básico-oportunidades.

\section{Análisis de la dimensión e-sectores}

La distribución muestra que los aspectos de e-business/e-commerce, e-cultura, gobierno electrónico y e-learning cubren el $67 \%$ de todas las variables/indicadores. El tema titulado "otras áreas" recoge las variables/indicadores en áreas como el periodismo en línea, la agricultura, deportes, etc., y tienen el 10\% de las variables/indicadores. Aspectos tales como e-ciencia y e-turismo tienen cada uno el 2\% (Figura 7).

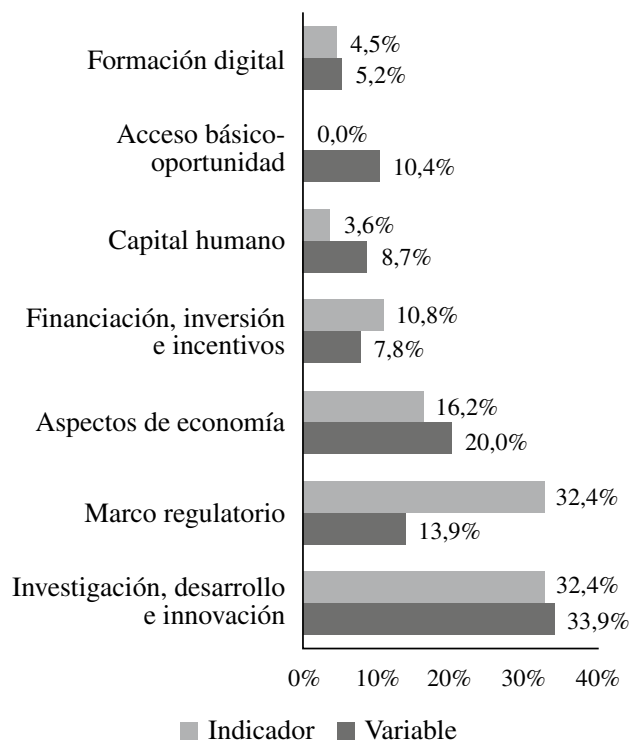

Figura 6. Distribución de variables e indicadores en los aspectos de la dimensión contexto.

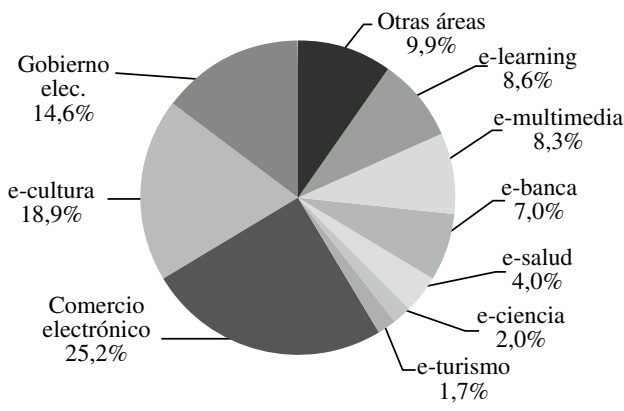

Figura 7. Distribución de las variables e indicadores en los aspectos de la dimensión e-sector.

El estudio demostró que la dimensión se compone en un $47 \%$ de las variables y el $53 \%$ de indicadores. En detalle se encontró que el $82,4 \%$ de las variables se distribuyen en los siguientes aspectos: $23,9 \%$ en comercio electrónico; $10,6 \%$ en e-cultura; $15,5 \%$ en gobierno electrónico; $11,3 \%$ en otras áreas; $10,6 \%$ en e-multimedia y $10,6 \%$ en e-banca. El restante $17,6 \%$ se distribuye en los aspectos de e-learning $(5,6 \%)$, e-salud $(4,9 \%)$, e-ciencia $(4,2 \%)$ y e-turismo $(2,8 \%)$.

En relación con la distribución de los indicadores, se encontró que el 77,5\% de ellos se agrupa en cuatro aspectos: comercio electrónico (26,3\%), e-cultura $(26,3 \%)$, gobierno electrónico $(13,8 \%)$ y e-learning $(11,3 \%)$. El restante $22,5 \%$ se distribuye en otras 
áreas $(8,8 \%)$, e-multimedia $(6,3 \%)$, e-banca $(3,8 \%)$, e-salud $(3,1 \%)$ y en e-turismo $(0,6 \%)$. Se encontró que el único aspecto que no tiene relacionados indicadores es e-ciencia. En la Figura 8 se presenta la distribución de las variables e indicadores en cada uno de los aspectos que la conforman.

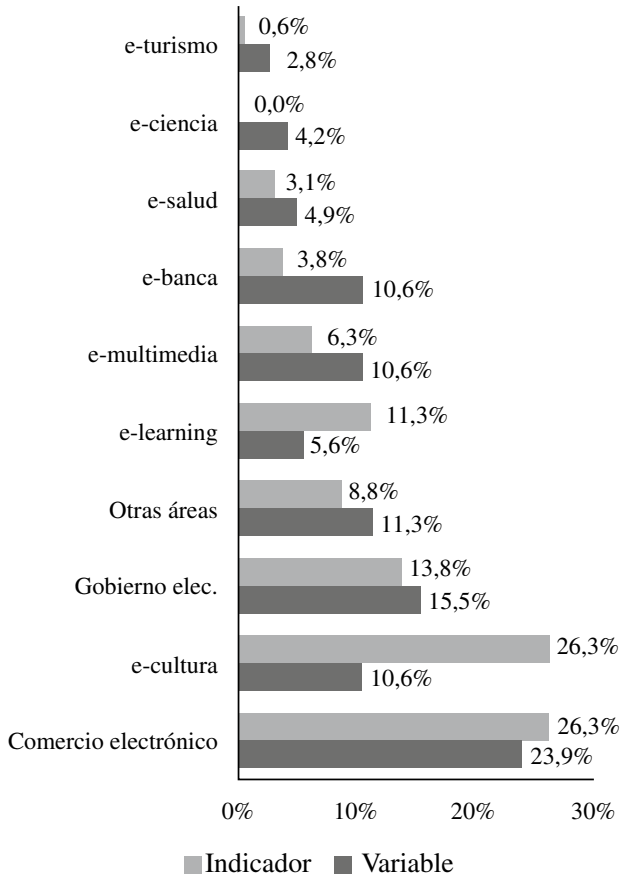

Figura 8. Distribución de variables e indicadores en los aspectos de la dimensión e-sectores

\section{Análisis de la dimensión participación en la SI \\ En este análisis se trabajó con el 3,1\% del total de variables/indicadores. Se encontró que estos aspectos se distribuyen como sigue: e-inclusión tiene $39 \%$ de las variables/indicadores de la dimensión de estudio. En cuanto a la brecha digital, e-accesibilidad y e-habilidades tienen asociados $12,9 \%, 22,6 \%$ y $25,8 \%$ de las variables / indicadores, respectivamente. En general, la dimensión se compone de $68 \%$ de las variables y $32 \%$ de los indicadores. En la Figura 9 se puede apreciar la distribución de los aspectos de la dimensión participación en la SI.}

En relación con la distribución de las variables que componen la dimensión, se encontró que más de la mitad se agrupan en el aspecto de e-inclusión $(52,4 \%)$, el restante $47,6 \%$ se distribuye entre los aspectos de e-habilidades $(28,6 \%)$ y en e-accesibilidad (19\%). El aspecto de brecha digital no tiene ninguna variable asociada. Los indicadores se encuentran

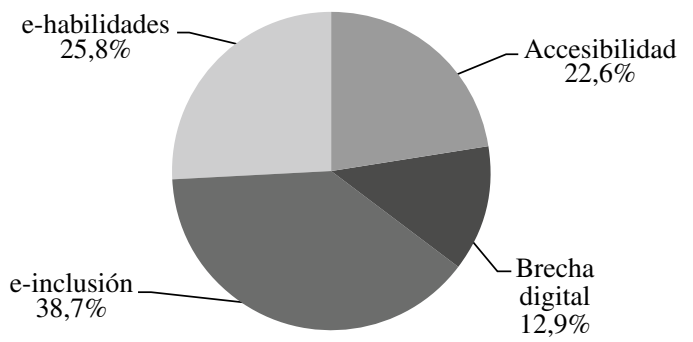

Figura 9. Distribución de variables/indicadores en los aspectos de la dimensión participación en la SI

distribuidos así: e-inclusión y e-habilidades cada una con 10\%; y e-accesibilidad y brecha digital, cada una con $40 \%$. En la Figura 10 se presenta la distribución de variables e indicadores en los aspectos de la dimensión participación en la SI.

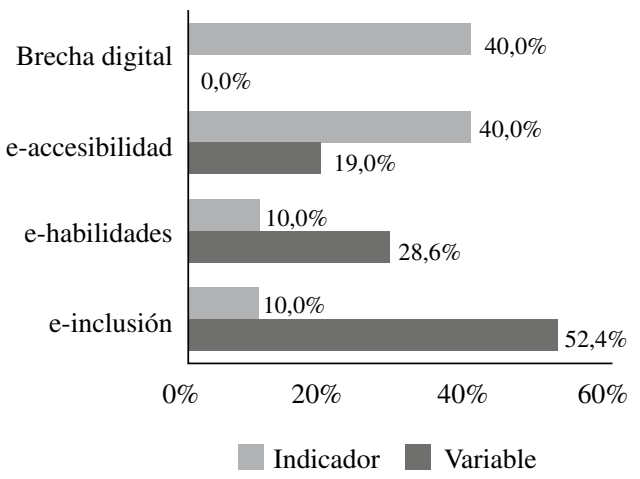

Figura 10. Distribución de variables e indicadores en los aspectos de la dimensión participación en la SI

\section{Análisis de la dimensión uso}

La dimensión uso se compone de 39,2\% de variables y $60,8 \%$ de indicadores. Estos corresponden al $7 \%$ del total de variables/indicadores identificados en el estudio.

\section{DISCUSIÓN DE RESULTADOS}

De acuerdo con los resultados, la dimensión más evaluada fue la de infraestructura, la cual agrupa $37,7 \%$ del total de variables/indicadores. En contraposición, la dimensión de participación en la SI fue la menos evaluada y tenía el menor número de variables / indicadores asociados $(3,1 \%)$. Lo anterior se puede entender si se considera, de un lado, que los aspectos de infraestructura son esenciales para el desarrollo de SI, y de otro, que evaluar la existencia 
o no de la infraestructura es mucho más sencillo que evaluar y verificar los aspectos relacionados con la inclusión digital y las habilidades en materia digital. Asimismo, la evaluación de la existencia o no de infraestructura es el parámetro empleado para la medición de brecha digital [5].

Al revisar los sistemas de evaluación seleccionados en relación con la cantidad de variables/indicadores, se detectó que el sistema e-España es el que ha desarrollado la mayor cantidad variables/indicadores, asimismo, es el que considera el mayor número de e-sectores. Este sistema tiene el 21,8\% del total de variables e indicadores bajo estudio, los cuales se distribuyen en todas las dimensiones en estudio. Estas características hacen del sistema eEspaña una referencia esencial en el proceso de benchmarking. Del mismo modo, los sistemas como Europe's Digital Competitiveness Report Main achievements of the i2010 strategy 2005-2009 e i2010 Information Space Innovation \& Investment in $R \& D$ Inclusion proponen variables/indicadores en todas las dimensiones. Por otra parte, se detectó que los sistemas Índice de Oportunidad Digital (DOI) e índice de acceso digital (DAI) se centran en evaluar aspectos de infraestructura y contexto.

El grado de desarrollo de las variables e indicadores se puede ver reflejado en la cantidad que han sido propuestos en cada uno de los aspectos objeto de estudio. En este sentido, se aprecia que los aspectos relacionados con la infraestructura como lo son: elementos y servicios físicos, servicios digitales, servicios relacionados y el sector de las TIC son los que tienen un mayor desarrollo $(37,7 \%)$ frente al desarrollo que se ha dado en los aspectos relacionados con la participación en la SI $(3,1 \%)$. En concreto, los elementos y servicios físicos es el aspecto con mayor número de variables (50) e indicadores definidos (144). Es importante destacar el creciente interés por pasar de una evaluación centrada en la brecha digital a una evaluación orientada a medir la participación de los ciudadanos, con lo cual se podrán apreciar nuevas propuestas de medición que equilibren la medición de los aspectos de infraestructura y los aspectos de participación [13-14].

En un segundo lugar, el análisis mostró que el 71,42\% de los sistemas en estudio evalúan aspectos relacionados con los e-sectores $(29,9 \%$ de variables/indicadores totales). El aspecto más evaluado es el comercio electrónico, lo cual puede entenderse si se considera que este sector fue uno de los primeros en contar con apoyo de las TIC, además de tener un impacto directo en la economía; pero deja la inquietud acerca de la evaluación que se hace para medir el progreso de la SI. Por otra parte, se detectó que en áreas como el turismo, la salud, la ciencia, la banca había un número importante de variables e indicadores, lo cual puede ser interpretado como un interés creciente en analizar el impacto de la TIC en los distintos sectores de la sociedad. Los resultados obtenidos en este análisis son similares a los obtenidos por [16] en su trabajo de análisis enfocado en el e-learning y el e-banking.

Acerca de la dimensión contexto, el estudio muestra que aspectos como el marco regulatorio; la economía; investigación, desarrollo e innovación; y financiación, inversión e incentivos agrupan el $83,6 \%$ del total de las variables/indicadores de esta dimensión. Esto puede ser entendido como la necesidad de verificar si los países han creado las condiciones necesarias para el desarrollo de la SI, asimismo, la evaluación de estos aspectos puede ser tomada para hacer seguimiento a los compromisos establecidos en convenios supranacionales y regionales. De igual manera, se detectó que es importante considerar al capital humano y su formación digital en la evaluación del avance de la SI, dado que es un actor imprescindible de ésta y dichos elementos son esenciales para garantizar su participación.

Relacionado con la dimensión de participación en la SI se encontró que está compuesta en su mayoría por variables (68\%). Lo cual puede evidenciar que la medición de la participación de los ciudadanos aún se está definiendo. En el caso específico del aspecto de e-inclusión se encontró que está compuesto casi en su totalidad de variables $(91,7 \%)$, lo cual puede deberse a que no se ha llegado a un consenso sobre qué medir. En contraposición se encontró que el aspecto de brecha digital está compuesto en su totalidad por indicadores, lo cual puede deberse a que este aspecto ha sido conceptualizado por varios autores y está estrechamente ligado a la existencia de infraestructura.

Los aspectos de e-habilidades y e-accesibilidad son considerados en la evaluación del avance de la SI, lo cual evidencia la importancia de considerar los desarrollos de la TIC para que sea accesible a toda la población independiente de su condición, y de la 
necesidad de formar en aspectos relacionados con el uso de la tecnología. En relación a la cantidad de variables e indicadores se encontró que estos dos aspectos tienen un comportamiento similar.

\section{CONCLUSIONES}

La investigación permitió evidenciar que la medición del avance de la SI se ha enfocado en la medición de la existencia de infraestructura, lo cual se explica al ser esta un elemento esencial para que se desarrolle la SI. De igual manera, se mostró que la evaluación de aspectos relacionados con los marcos regulatorio; la financiación, inversión e incentivos y la investigación, desarrollo e innovación, son considerados en la evaluación de la SI como elementos que viabilizan y posibilitan el generar la SI como modelo de sociedad.

Por otra parte, se detectó que la evaluación de las e-sectores se ha ampliado a áreas como: la ciencia, el turismo, la agricultura, etc., lo cual pone de manifiesto el interés por evaluar el uso de las TIC en los diversos sectores de la sociedad. En relación con los aspectos relacionados con la participación en la SI se encontró que su desarrollo es aún incipiente y que se debe trabajar en definir qué medir, para así obtener indicadores que permitan medir el desarrollo de la SI desde la perspectiva del usuario.

Por otra parte, la metodología empleada permitió analizar la cantidad de variables e indicadores que se han desarrollado en cada uno de los aspectos propuestos para medir la SI, lo cual permite inferir el grado de desarrollo de estos y entender cuáles son los aspectos más evaluados. Es importante mencionar que el trabajo de administración de los datos y generación de los reportes fue posible gracias al uso de la herramienta software desarrollada para tal fin.

En cuanto a las dificultades de la investigación se encontró que el acceso a la información fue una de las limitantes más significativas. En relación con el trabajo futuro, se espera profundizar en cada uno de los aspectos de las dimensiones bajo estudio en el marco del desarrollo del proyecto e-métrica Colombia [15].

Finalmente, se concluye que el estudio realizado permite obtener un mayor grado de profundidad y entendimiento acerca de los aspectos que son considerados para medir el avance de la incorporación de la SI como modelo de sociedad, y de la participación de los ciudadanos en la misma, así como poner en evidencia que las variables e indicadores empleados determinan una clasificación entre las entidades evaluadas, pero no permiten identificar el estado de madurez de la SI.

\section{AGRADECIMIENTOS}

Las autoras expresan sus agradecimientos al Departamento Nacional de Ciencia y Tecnología de Colombia-COLCIENCIAS, a la Universidad del Magdalena.

\section{REFERENCIAS}

[1] J.M. Sánchez-Torres. "Propuesta metodológica para evaluar políticas públicas de promoción del e-government como campo de aplicación de la sociedad de la información. El caso colombiano". Tesis para optar al grado de doctor. Universidad Autónoma de Madrid. Madrid, España. 2006.

[2] J.E. Katz y R.E. Rice. "Social consequences of Internet use: access, involvement and interaction. Estados Unidos de América". MIT Press. 2002.

[3] M.P. González-Zabala y J.M. Sánchez-Torres. "La Sociedad de la Información: Génesis, Iniciativas, Concepto y Modelos". Revista UIS Ingenierías. En evaluación. 2011. ISSN: 1657-4583.

[4] eLAC. "II Conferencia Ministerial sobre la Sociedad de la Información". II Conferencia Ministerial sobre la Sociedad de la Información de América Latina y el Caribe. San Salvador, El Salvador. 6-8 de noviembre de 2007.

[5] European Commission. "Communication European i2010 initiative on e-Inclusion -to be part of the information society". 2008. URL: http://ec.europa.eu/information_society/ activities/einclusion/bepartofit/overview/ index_en.htm. Fecha de consulta: 9 de junio de 2010.

[6] eLAC. "Plan de Acción sobre la Sociedad de la Información de América Latina y el Caribe eLAC2007". 2007. URL: www.cudi. mx/gobernanza/CMSI_plan_eLAC\%202007. pdf. Fecha de consulta: 17 de mayo de 2010. 
[7] UTI y Naciones Unidas. "World Summit on the Information Society". Ginebra, Suiza. 10 -12 de diciembre de 2003. URL: http://www. itu.int/wsis/index.html. Fecha de consulta: 16 de junio de 2010.

[8] CMSI. "Declaración de Principios de Ginebra". 2003. URL: http://www.itu.int/ wsis/docs/geneva/official/dop-es.html. Fecha de consulta: 15 de marzo de 2010.

[9] CMSI-Cumbre Mundial para la Sociedad de la Información. "Agenda de Túnez para la Sociedad de la Información". Túnez, República Tunecina. Junio 2006. URL: www.itu.int/wsis/docs2/tunis/off/6rev1-es. html. Fecha de consulta: 20 de noviembre de 2009.

[10] CMSI-Cumbre Mundial para la Sociedad de la Información. "Compromiso de Túnez". Túnez, República Tunecina. Junio 2006. URL: www.itu.int/wsis/docs2/tunis/off/7-es. html. Fecha de consulta: 20 de noviembre de 2009.

[11] C. Bianco, G. Lugones, F. Peirano y M. Salazar. "Indicadores de la Sociedad del Conocimiento: Aspectos conceptuales y metodológicos". Paper presentado en el segundo taller de Indicadores de Sociedad de la Información. Lisboa, Portugal. Febrero de 2003.

[12] G. Lugones, C. Bianco, F. Peirano y M. Salazar. "Indicadores de la Sociedad del Conocimiento e Indicadores de Innovación. Vinculaciones e Implicancias Conceptuales y Metodológicas". En Nuevas Tecnologías de Información y Comunicación. Miño y Dávila, pp. 141-171. Buenos Aires, Argentina. 2003.

[13] M.P. González-Zabala and J.M. SánchezTorres. "Design on Evaluation Model for Social Inclusion in the Information Society: A proposal from action research approach". 5th Euro American Conference on Telematics and Information Systems. Ciudad de Panamá, Panamá. 2010.

[14] M.P. González-Zabala y J.M. Sánchez-Torres. "Propuesta de un modelo para evaluar la e-inclusión. El caso colombiano". Propuesta de tesis doctoral. Facultad de Ingeniería. Universidad Nacional de Colombia. Bogotá, Colombia. 2010.

[15] J.M. Sánchez-Torres. "e-Métrica ColombiaFase I". Código: 9350. Convocatoria Nacional de Investigación 2009. Modalidad IV: Apoyo dirigido a nuevos docentes investigadores de la Universidad Nacional de Colombia a través de Proyectos. Universidad Nacional de Colombia. Bogotá, Colombia. 2009.

[16] A. Bustamante y J.M. Sánchez-Torres. "Indicadores de la Sociedad de la Información para la medición en e-banking y e-learning". Encuentro Nacional de Investigación en Posgrados. Universidad Nacional de Colombia. Bogotá, Colombia. 2009.

[17] Knowledge for Development. "KI and KEI Indexes". 1995. URL: http://web. worldbank.org/WBSITE/EXTERNAL/ WBI/WBIPROGRAMS/KFDLP/EXTUN IKAM/0,,contentMDK:20584278 menuP K:1433216 pagePK:64168445 piPK:641 68309 theSitePK:1414721,00.html. Fecha de Consulta: 11 de abril de 2010.

[18] M.P. González-Zabala y J.M. Sánchez-Torres. "Modelo Conceptual de la Sociedad de la Información como Sistema de Actividad Humana". Mimeo. Universidad Nacional de Colombia. Bogotá, Colombia. 2010.

[19] M.P. González-Zabala, E.A. Galvis y J.M. Sánchez-Torres. "Diseño de un Sistema de Información para el Análisis de Sistemas de Medición de la Sociedad de la Información". Revista UIS Ingenierías-En evaluación. 2011. ISSN: 1657-4583.

[20] Commission of the European Communities. "i2010-Annual Information Society Report 2007”. Bruselas, Bélgica. 2007. URL: http://ec.europa.eu/information_society/ eeurope/i2010/docs/annual_report/2007/ sec_2007_395_en_documentdetravail3_p. pdf. Fecha de Consulta: 13 de mayo de 2010.

[21] European Commission Information Society and Media. "i2010-Annual Information Society Report 2007-A European Information Society for Growth and Employment". Office for Official Publications of the European Communities. Italia. 2007. URL: http://ec.europa.eu/information_society/ eeurope/i2010/docs/annual_report/2007/ i2010_ar_2007_en.pdf. Fecha de Consulta: 15 de mayo de 2010.

[22] Commission of the European Communities. "i2010-First Annual Report on the European Information Society”. Bruselas, Bélgica. 2006. URL: http://ec.europa.eu/ 
information_society/eeurope/i2010/docs/ annual_report/2006/sec_2006_604_en.pdf. Fecha de Consulta: 16 de mayo de 2010.

[23] Commission of the European Communities. "Volume 1: i2010-Annual Information Society Report 2008 Benchmarking i2010: Progress and Fragmentation in the European Information Society". Buselas, Bélgica 2008. URL: http://ec.europa.eu/ information_society/eeurope/i2010/docs/ annual_report/2008/sec_2008_470_Vol_1. pdf. Fecha de Consulta: 18 de mayo de 2010.

[24] L. Waverman, K. Dasgupta, y E. van del Merwe. "The Connectivity ScorecardInforme de 2010". LECG-Nokia Siemens Networks, 2010. URL: http://www. connectivityscorecard.org/images/uploads/ media/TheConnectivityReport2010.pdf. Fecha de Consulta: 12 de febrero de 2010.

[25] L. Waverman, K. Dasgupta and N. Brooks. "The Connectivity Scorecard-Informe de 2009". LECG-Nokia Siemens Networks. 2009. URL: http://www.connectivityscorecard.org/images/ uploads/media/TheConnectivityReport2009. pdf. Fecha de Consulta: 12 de febrero de 2010.

[26] L. Waverman, K. Dasgupta and J. Tonkin. "The Connectivity Scorecard-Informe de 2008". LECG-Nokia Siemens Networks, Bruselas, Bélgica. 2008. URL: http://ec.europa.eu/ information_society/eeurope/i2010/docs/ annual_report/2008/sec_2008_470_Vol_1. pdf. Fecha de Consulta: 12 de febrero de 2010.

[27] ITU, UNCTAD and World Summit on the Information Society. "World Information Society Report 2007-Beyond WSIS". Ginebra, Suiza. 2007. URL: http://www.itu.int/osg/spu/ publications/worldinformationsociety/2007/ WISR07_full-free.pdf. Fecha de Consulta: 9 de abril de 2010.

[28] ITU, KADO, UNCTAD and MIC. "World Information Society Report 2006". Julio de 2006. URL: http://www.ifap.ru/library/ book084.pdf. Fecha de Consulta: 10 de abril de 2010.

[29] Fundación Orange. “eEspaña 2009-Informe anual sobre el desarrollo de la sociedad de la información en España". Fundación Orange. España. 2009. URL: http://www. fundacionorange.es/areas/25_publicaciones/ e2009.pdf. Fecha de Consulta: 10 de febrero de 2010.

[30] Fundación Orange. “eEspaña 2008-Informe anual sobre el desarrollo de la sociedad de la información en España”. Fundación Orange. España. 2008. URL: http://www. fundacionorange.es/areas/25_publicaciones/ e2008.pdf. Fecha de Consulta: 13 de febrero de 2010.

[31] Fundación Orange. "eEspaña 2007-Informe anual sobre el desarrollo de la sociedad de la información en España". Fundación Orange. España. 2007. URL: http://www. fundacionorange.es/areas/25_publicaciones/ e2007.pdf. Fecha de Consulta: 24 de febrero de 2010.

[32] Fundación Orange. “eEspaña 2006-Informe anual sobre el desarrollo de la sociedad de la información en España". Fundación Orange, España. 2006. URL: http://www. fundacionorange.es/areas/25_publicaciones/ eEspana_2006.pdf. Fecha de Consulta: 15 de febrero de 2010.

[33] Fundación Orange. "eEspaña 2005-Informe anual sobre el desarrollo de la sociedad de la información en España". Fundación Orange. España. 2005. URL: http://www. fundacionorange.es/areas/25_publicaciones/ EESPA_A2005_COMPLETO_V3.pdf.Fecha de Consulta: 14 de febrero de 2010.

[34] Fundación Orange. "eEspaña 2004-Informe anual sobre el desarrollo de la sociedad de la información en España". Fundación Orange. España. 2004. URL: http://www. fundacionorange.es/areas/25_publicaciones/ publi_251_4_2.asp. Fecha de Consulta: 14 de febrero de 2010.

[35] Fundación Orange. “eEspaña 2003-Informe anual sobre el desarrollo de la sociedad de la información en España”. Fundación Orange. España. 2003. URL: http://www. fundacionorange.es/areas/25_publicaciones/ publi_251_3_2.asp. Fecha de Consulta: 15 de febrero de 2010.

[36] Fundación Orange. "eEspaña 2002-Informe anual sobre el desarrollo de la sociedad de la información en España”. Fundación Orange. España. 2002. URL: http://www. fundacionorange.es/areas/25_publicaciones/ publi_251_2.asp. Fecha de Consulta: 15 de febrero de 2010. 
[37] Fundación Orange. “eEspaña 2001-Informe anual sobre el desarrollo de la sociedad de la información en España”. Fundación Orange. España. 2001. URL: http://www. fundacionorange.es/areas/25_publicaciones/ publi_251_1.asp. Fecha de Consulta: 18 de febrero de 2010.

[38] European Commission. Europe's Digital Competitiveness Report-Main achievements of the i2010 strategy 2005-2009. ISBN: 978-92-79-12823-3. Luxembourg: European Communities, 2009. URL: http://ec.europa. eu/information_society/eeurope/i2010/docs/ annual_report/2009/digital_competitiveness. pdf. Fecha de Consulta: 14 de abril de 2010.

[39] ESIS Project Management Support Team. "European Survey of Information SocietyExtension to Central and Eastern European Countries and the Mediterranean Area". 2000. URL: http://kbn.icm.edu.pl/is2000/ pdf/word6.PDF Fecha de Consulta: 8 de febrero de 2010.

[40] ESIS Project Management Support Team. "ESIS -Information Society indicators in the Member States of the European Union". Octubre 2000. URL: http://banners. noticiasdot.com/termometro/boletines/docs/ paises/europa/ue/1999/ispo/ue_ispo_ESIS_ indicators_2000.pdf. Fecha de Consulta: 8 de febrero de 2010.

[41] IDC. "IDC's Informational Society Index". 2004. URL: http://www.idc.com/groups/isi/ main.html. Fecha de Consulta: 10 de abril de 2010.

[42] S. Minton and C.M. Glasheen. "IDCInformation Society Index 2009”. 2009. URL: http://www.idc.com/groups/isi/main. html. Fecha de Consulta: 29 de abril de 2010.

[43] S. Minton, C.M. Glasheen and A. Toncheva. "IDC-Information Society Index 2008-2013 Forecast". 2009. URL: http://www.idc.com/ groups/isi/main.html. Fecha de Consulta: 29 de abril de 2010.

[44] OSILAC. "Indicadores de TIC". 2005. URL: http://websie.eclac.cl/sisgen/ ConsultaIntegrada.asp?idAplicacion $=16$ \&idioma=. Fecha de Consulta: 25 de mayo de 2010.

[45] PNUD-Programa de las Naciones Unidas para el Desarrollo. "Índice de Adelanto Tecnológico". 2001. URL: http://www.oei.
es/catmexico/Indice_Adelanto_Tecnologico_ PNUD.pdf. Fecha de Consulta: 12 de abril de 2010.

[46] UNCTAD. "Information and Communication Technology Development Indices". 2003. URL: http://www.unctad.org/Templates/ webflyer.asp?docid=3397\&intItemID=139 $7 \&$ lang=1. Fecha de Consulta: 29 de agosto de 2010.

[47] UNCTAD. "Information economy report 2007-2008". Naciones Unidas, 2007. URL: http://www.unctad.org/en/docs/ sdteecb20071_en.pdf. Fecha de Consulta: 14 de abril de 2010.

[48] OECD. "OECD Key ICT Indicators". 2009. URL: http://www.oecd.org/document/23/0,3 343,en_2649_34449_33987543_1_1_1_1,00. html. Fecha de Consulta: 10 de abril de 2010.

[49] OSILAC. "ICT STATISTICAL INFORMATION SYSTEM”. 2007. URL: http://www.cepal.org/tic/flash/. Fecha de Consulta: 29 de abril de 2010.

[50] R.D. Atkinson and R. Coduri. "THE 2002 STATE NEW ECONOMY INDEXBenchmarking Economic Transformation in the States". Progressive Policy Institute. 2002. URL: http://inpathways.net/ppi_state_ index_2002.pdf. Fecha de Consulta: 3 de diciembre de 2010.

[51] R. Atkinson and R. Court. "The state new economy index-Benchmarking Economic Transformation in the States". Progressive Policy Institute. 1999. URL: http://www. research.fsu.edu/techtransfer/documents/ snei.pdf. Fecha de consulta: 3 de diciembre de 2009.

[52] ITU. "Digital Access Index: World's First Global ICT Ranking”. 2003. URL: http://www.itu.int/newsroom/press_ releases/2003/30.html. Fecha de Consulta: 10 de abril de 2010.

[53] i2010 High Level Group. "Benchmarking Digital Europe 2011-2015-a conceptual framework". Octubre 2009. URL: http:// ec.europa.eu/information_society/eeurope/ i2010/docs/benchmarking/benchmarking digital_europe_2011-2015.pdf. Fecha de Consulta: 29 de agosto de 2010.

[54] OECD. "Guide to measuring the information society, 2009". Secretary-General of the OECD. 2009. URL: http://www.oecd.org/ 
dataoecd/25/52/43281062.pdf. Fecha de Consulta: 24 de mayo de 2010.

[55] OECD. "Working Party on Indicators for the Information Society-Guide to Measuring the Information Society". Secretary-General of the OECD, 2005. URL: http://www.oecd. org/dataoecd/41/12/36177203.pdf. Fecha de Consulta: 28 de mayo de 2010.

[56] i2010 High Level Group. "i2010 Information Space Innovation \& Investment in R\&D Inclusion-Benchmarking Framework”. 2006. URL: http://ec.europa.eu/information_society/ eeurope/i2010/docs/high_level_group/ i2010_benchmarking_framework.pdf. Fecha de Consulta: 17 de mayo de 2010.

[57] Naciones Unidas. "Indicadores clave de las tecnologías de la información y de las comunicaciones". 2005. URL: http://www. cepal.org/cgi-bin/getProd.asp?xml=/socinfo/ noticias/documentosdetrabajo/7/23117/ P23117.xml\&xsl=/socinfo/tpl/p38f. xsl\&base=/socinfo/tpl/top-bottom.xsl. Fecha de Consulta: 23 de abril de 2010.
[58] S. Roberts, E. Magpantay, V. Gray, S. Teltscher, S. Fondeur, D. Korka, M. Schaaper, C. Akpabie, G. Boade and S. Ellis. "The Global Information Society: a Statistical View". ITU, OECD, UNESCO, EUROSTAT, UNITED NATIONS, BM, UNCTAD, ESCWA, ELAC, ECONOMIC COMMISSION FOR AFRICA, UNITED NATIONS ESCAP. 2008. URL: http://www. eclac.org/socinfo/noticias/noticias/2/33492/ Globalinformation.pdf. Fecha de Consulta: 18 de abril de 2010.

[59] J.M. Sánchez-Torres, M.P. González-Zabala y M. Salazar. "Indicadores de e-inclusión en el Marco de los Sistemas de Evaluación de la Sociedad de la Información". VIII Congreso Ibero-Americano de Indicadores de Ciencia y Tecnología. Madrid, España. 2010. Pág. 20.

[60] M.P. González-Zabala y J.M. Sánchez-Torres. "Métrica de la Sociedad de la InformaciónInforme". Mimeo, Universidad Nacional de Colombia. Bogotá, Colombia. 2010. 